\title{
CP violation in extended Higgs sectors
}

\section{O. M. Ogreid}

Western Norway University of Applied Sciences,

Postboks 7030, N-5020 Bergen, Norway,

E-mail: omo@hvl.no

\section{P. Osland*}

Department of Physics and Technology, University of Bergen,

Postboks 7803, N-5020 Bergen, Norway,

E-mail: Per.Oslandeuib.no

\section{N. Rebelo}

Centro de Física Teórica de Partículas - CFTP and Dept de Física

Instituto Superior Técnico - IST, Universidade de Lisboa, Av. Rovisco Pais,

P-1049-001 Lisboa, Portugal

E-mail: rebelo@tecnico.ulisboa.pt

We discuss how one can identify $\mathrm{CP}$ violation (and conservation) in multi-Higgs-doublet potentials. After a brief review of $\mathrm{CP}$ violation in the $2 \mathrm{HDM}$, we refer to the fact that for NHDM with $N \geq 3$ the well known methods useful in the case $N=2$ have not been generalized in order to provide a set of well defined necessary and sufficient conditions for $\mathrm{CP}$ conservation. We then present a simple method, proposed by the authors, to be used in such cases. Two non-trivial examples based on an $S_{3}$-symmetric three-doublet model are analyzed by means of this new method.

Corfu Summer Institute 2017 'School and Workshops on Elementary Particle Physics and Gravity' 2-28 September 2017

Corfu, Greece

${ }^{*}$ Speaker. 


\section{Introduction}

Multi-Higgs-doublet models provide a rich framework for Beyond-Standard-Model physics. Models with two doublets (2HDM) have long received a lot of attention [1, 2]. Models with three Higgs doublets are also well motivated and for a recent review of such models see, for instance, Ref. [3]. As the number of doublets increases, so does the number of free parameters. Symmetries play an important rôle in reducing this number, thus adding predictive power to the models.

The most general NHDM potential is given by

$$
V=Y_{a b} \Phi_{a}^{\dagger} \Phi_{b}+\frac{1}{2} Z_{a b c d}\left(\Phi_{a}^{\dagger} \Phi_{b}\right)\left(\Phi_{c}^{\dagger} \Phi_{d}\right)
$$

where the $\Phi$ are $\mathrm{SU}(2)$ doublets and $a, b, c, d$ run over the values 1 to $N$, with repeated indices to be summed over. For $N=3$ the potential has 3 diagonal bilinear terms (real): $Y_{11}, Y_{22}, Y_{33}$ and 3 off-diagonal ones (complex) satisfying $Y_{b a}^{*}=Y_{a b}$. Furthermore, there are many $Z_{a b c d}$, some real, some complex, satisfying:

$$
Z_{b a d c}^{*}=Z_{a b c d}, \quad Z_{c d a b}=Z_{a b c d} .
$$

All counted, there are 54 parameters, but they are not all independent, since we may rotate:

$$
\left(\begin{array}{l}
\Phi_{1}^{\prime} \\
\Phi_{2}^{\prime} \\
\Phi_{3}^{\prime}
\end{array}\right)=U\left(\begin{array}{l}
\Phi_{1} \\
\Phi_{2} \\
\Phi_{3}
\end{array}\right)
$$

with $U$ an arbitrary unitary matrix. This way, one may diagonalize the bilinear part (removing 6 parameters) and also remove 2 relative phases between the $\Phi$. The remaining number of linearly independent parameters is thus 46 [4]. For comparison, the number of independent parameters in the $2 \mathrm{HDM}$ is 11 . This illustrates the fact that the number of free parameters in multi-Higgs-doublet models grows fast with the number of doublets, thus leading to a very rich structure. Without further constraints these models may lead, for instance, to potentially dangerous flavour changing neutral currents in the quark sector, and may also lead to other new phenomena already ruled out by experiment.

We will discuss methods for identifying $\mathrm{CP}$ violation or conservation, in $N \geq 3$ models, and will illustrate their power by applying them to an $S_{3}$-symmetric, ten-parameter $N=3$ potential. These methods can equally well be applied to the case of $N=2$. However, as will be explained in section 3 , this case is simple and has already been well studied.

\section{Identifying $\mathrm{CP}$ violation}

$\mathrm{CP}$ violation in gauge theories requires the introduction of a scalar sector. Pure gauge theories including fermions cannot violate CP [5].

If all coefficients in the potential and all vacuum expectation values (vevs) are real, then CP is conserved. However, the converse does not always hold. For instance, it is possible to have a complex potential that does not violate $\mathrm{CP}$ explicitly, and it is also possible to have explicit $\mathrm{CP}$ 
conservation with a real potential together with non-trivial complex vevs that do not violate $\mathrm{CP}$ spontaneously.

The most general CP transformation for multi-Higgs models is given by [6, 7]:

$$
\Phi_{i} \stackrel{\mathrm{CP}}{\longrightarrow} W_{i j} \Phi_{j}^{*}
$$

for $W$ a unitary, arbitrary transformation. Eq. (2.1) is a combination of the CP transformation of each single Higgs doublet with a Higgs basis transformation. This, or in alternative the fact that physics does not change with a change of Higgs basis, has been exploited in the identification of $\mathrm{CP}$-odd re-parametrization invariants sensitive to explicit $\mathrm{CP}$ violation $[8,9]$. For the 2HDM these conditions are equivalent to conditions written in terms of the charged-Higgs mass and its quartic coupling [10]. If all coefficients of the potential are real, then there is no explicit CP violation. Assuming that the potential is real, there is still the possibility of having spontaneous $\mathrm{CP}$ violation through phases appearing in the vevs. CP violation can only be considered to be spontaneous if there is explicit $\mathrm{CP}$ conservation.

In the case of the $2 \mathrm{HDM}$ a full set of invariant conditions sensitive to spontaneous $\mathrm{CP}$ violation involving the potential and the vevs has been derived [11, 12]. For the 2HDM, Higgs-basis-invariant conditions can be expressed in terms of masses and couplings [11, 12, 13, 14]. The technique to generate such invariants can be applied in theories with more than two Higgs doublets. However, a full set of necessary and sufficient conditions for $\mathrm{CP}$ conservation in the cases of $N=3$ or higher has not yet been identified. There are examples in the literature for special cases with particular symmetries $[15,16]$. Higgs basis invariants have also been applied to determine tree level Higgs couplings and masses without putting the emphasis on CP violation [17].

An alternative method to determine whether or not there is spontaneous CP violation in an NHDM was provided in Ref. [6] where it was shown that if there is a symmetry $U$ of the Lagrangian, acting on the Higgs doublets, $\mathscr{L}(U \Phi)=\mathscr{L}(\Phi)$, under which the Higgs vevs satisfy the relation:

$$
U_{i j}\left\langle 0\left|\Phi_{j}\right| 0\right\rangle^{*}=\left\langle 0\left|\Phi_{i}\right| 0\right\rangle
$$

then the vacuum is invariant and there is no spontaneous $\mathrm{CP}$ violation. This is a very powerful relation but in some cases finding the matrix $U$ that satisfies Eq. (2.2) may not be straightforward.

For more complicated cases a simple procedure to determine whether or not there is CP violation has been proposed in [18] and consists of starting by making a transformation to a Higgs basis where only one of the Higgs doublets acquires a vev different from zero, chosen to be real, and all other doublets have zero vevs $[19,20]$. The next step consists in using the freedom to rephase the doublets with zero vev or, if necessary, even to perform a $U(N-1)$ unitary rotation of these fields in order to make all the coefficients of the potential real. This method is sensitive to both explicit and spontaneous $\mathrm{CP}$ violation.

In section 4 we will show how this method can be used in the case of an $S_{3}$-symmetric, tenparameter $N=3$ potential, where the determination of the matrix $U$ of Eq. (2.2) is not straightforward.

\section{Review of CP violation in the $2 \mathrm{HDM}$}

The most general 2HDM contains three neutral physical scalars, but these need not be eigen- 
states of CP. This can be illustrated by going to the Higgs basis $[19,20]$ where the two Higgs doublets are parametrized as

$$
\Phi_{1}=\left(\begin{array}{c}
G^{+} \\
\left(v+\eta_{1}+i G_{0}\right) / \sqrt{2}
\end{array}\right), \quad \Phi_{2}=\left(\begin{array}{c}
H^{+} \\
\left(\eta_{2}+i \chi_{2}\right) / \sqrt{2}
\end{array}\right),
$$

and the potential takes the form

$$
\begin{aligned}
V\left(\Phi_{1}, \Phi_{2}\right) & =-\frac{1}{2}\left\{m_{11}^{2} \Phi_{1}^{\dagger} \Phi_{1}+m_{22}^{2} \Phi_{2}^{\dagger} \Phi_{2}+\left[m_{12}^{2} \Phi_{1}^{\dagger} \Phi_{2}+\text { h.c. }\right]\right\} \\
& +\frac{\lambda_{1}}{2}\left(\Phi_{1}^{\dagger} \Phi_{1}\right)^{2}+\frac{\lambda_{2}}{2}\left(\Phi_{2}^{\dagger} \Phi_{2}\right)^{2}+\lambda_{3}\left(\Phi_{1}^{\dagger} \Phi_{1}\right)\left(\Phi_{2}^{\dagger} \Phi_{2}\right)+\lambda_{4}\left(\Phi_{1}^{\dagger} \Phi_{2}\right)\left(\Phi_{2}^{\dagger} \Phi_{1}\right) \\
& +\frac{1}{2}\left[\lambda_{5}\left(\Phi_{1}^{\dagger} \Phi_{2}\right)^{2}+\text { h.c. }\right]+\left\{\left[\lambda_{6}\left(\Phi_{1}^{\dagger} \Phi_{1}\right)+\lambda_{7}\left(\Phi_{2}^{\dagger} \Phi_{2}\right)\right]\left(\Phi_{1}^{\dagger} \Phi_{2}\right)+\text { h.c. }\right\} .
\end{aligned}
$$

The parameters $m_{12}^{2}$ together with $\lambda_{5}, \lambda_{6}$ and $\lambda_{7}$ can be complex. The minimization conditions impose $m_{12}^{2}=v^{2} \lambda_{6}$. The mass-squared matrix will depend on the parameters $m_{22}^{2}, \lambda_{1}, \lambda_{3}, \lambda_{4}, \lambda_{5}$ and $\lambda_{6}$ :

$$
\mathscr{M}^{2}=\left(\begin{array}{ccc}
\lambda_{1} v^{2} & \operatorname{Re} \lambda_{6} v^{2} & -\operatorname{Im} \lambda_{6} v^{2} \\
\operatorname{Re} \lambda_{6} v^{2} & \frac{1}{2}\left(-m_{22}^{2}+\left(\lambda_{3}+\lambda_{4}+\operatorname{Re} \lambda_{5}\right) v^{2}\right) & -\frac{1}{2} \operatorname{Im} \lambda_{5} v^{2} \\
-\operatorname{Im} \lambda_{6} v^{2} & -\frac{1}{2} \operatorname{Im} \lambda_{5} v^{2} & \frac{1}{2}\left(-m_{22}^{2}+\left(\lambda_{3}+\lambda_{4}-\operatorname{Re} \lambda_{5}\right) v^{2}\right)
\end{array}\right) .
$$

Notice that the mass matrix does not include $\lambda_{7}$. If $\lambda_{5}$ and $\lambda_{6}$ can be made simultaneously real by a redefinition of $\Phi_{2}$ there is no mixing among CP-even and CP-odd fields. This is the case, for instance, if $\lambda_{6}=0$ or $\lambda_{5}=0$. In particular, for $\lambda_{6}=0$ we can make $\lambda_{5}$ real by rephasing $\Phi_{2}$ and the mass matrix becomes automatically diagonal. However, in order to conclude that CP is conserved one must check whether or not $\lambda_{7}$ can also be made real with the same rephasing of $\Phi_{2}$ that makes $\lambda_{5}$ and $\lambda_{6}$ real, otherwise there will be CP violation in the trilinear and quartic couplings.

These conditions will look different in a general (non-Higgs) basis, but the different possibilities of having $\mathrm{CP}$ conservation or violation can be sorted out by exploring the basis-transformation invariants mentioned above, see $[11,12]$ and $[8,9]$.

A different approach is to ask whether a basis exists in which the potential and the vevs are simultaneously real [23]. When applied in the Higgs basis [18] this constitutes a powerful test for the study of multi-Higgs-doublet models as illustrated in what follows.

\section{The use of the Higgs basis to test for CP Conservation}

\subsection{The 2HDM with real coefficients}

Without loss of generality, the $2 \mathrm{HDM}$ potential can be written as: ${ }^{1}$

$$
\begin{aligned}
V(\phi) & =-\lambda_{1} \phi_{1}^{\dagger} \phi_{1}-\lambda_{2} \phi_{2}^{\dagger} \phi_{2} \\
& +A\left(\phi_{1}^{\dagger} \phi_{1}\right)^{2}+B\left(\phi_{2}^{\dagger} \phi_{2}\right)^{2}+C\left(\phi_{1}^{\dagger} \phi_{1}\right)\left(\phi_{2}^{\dagger} \phi_{2}\right)+\bar{C}\left(\phi_{1}^{\dagger} \phi_{2}\right)\left(\phi_{2}^{\dagger} \phi_{1}\right) \\
& +\frac{1}{2}\left[\left(\phi_{1}^{\dagger} \phi_{2}\right)\left(D \phi_{1}^{\dagger} \phi_{2}+E \phi_{1}^{\dagger} \phi_{1}+F \phi_{2}^{\dagger} \phi_{2}\right)+\text { h.c. }\right],
\end{aligned}
$$

\footnotetext{
${ }^{1}$ Here, we follow the notation of ref. [21], the $\lambda_{1}$ and $\lambda_{2}$ should not be confused with those of Eq. (3.2).
} 
with the bilinear part already diagonal, making use of the freedom in the choice of Higgs basis as described in the Introduction. In general, the coefficients $D, E$ and $F$ can all be complex, but at least one of then can be made real by simply rephasing one of the doublets. In this way one ends up with the eleven independent real parameters mentioned before. There is explicit CP violation if it is not possible to make all three coefficients real at the same time.

It is well known that, even with real coefficients, the $2 \mathrm{HDM}$ can violate $\mathrm{CP}$ spontaneously [21], since, in this case the potential allows for non-real vevs: $\left(\rho_{1} e^{i \theta}, \rho_{2}\right)$. In the absence of an additional symmetry of the potencial and for a nontrivial phase, there is no way of verifying the condition given by Eq. (2.2). If a $Z_{2}$ symmetry is imposed on the $2 \mathrm{HDM}$ Lagrangian there is neither explicit nor spontaneous CP violation. The possibility of spontaneous CP violation in two-doublet models with a softly broken discrete symmetry was pointed out in [22].

Starting with the potential of the 2HDM in the notation of Eq. (4.1) and with real coefficients there is still the possibility of having spontaneous $\mathrm{CP}$ violation [21] since, as mentioned above, there is a region of parameters where the vevs are of the form $\left(\rho_{1} e^{i \theta}, \rho_{2}\right)$, with $\theta$ non trivial and both $\rho_{1}$ and $\rho_{2}$ different from zero.

The Higgs basis is reached via the transformation

$$
\left(\begin{array}{l}
\phi_{1}^{\prime} \\
\phi_{2}^{\prime}
\end{array}\right)=\frac{1}{v}\left(\begin{array}{cc}
1 & 0 \\
0 & e^{i \chi}
\end{array}\right)\left(\begin{array}{cc}
\rho_{1} & \rho_{2} \\
-\rho_{2} & \rho_{1}
\end{array}\right)\left(\begin{array}{cc}
e^{-i \theta} & 0 \\
0 & 1
\end{array}\right)\left(\begin{array}{l}
\phi_{1} \\
\phi_{2}
\end{array}\right)
$$

with (normalization) $v^{2}=\rho_{1}^{2}+\rho_{2}^{2}$. This basis is defined as the basis where the vevs are real and are of the form $(v, 0)$. In this basis CP violation manifests itself by the impossibility of making the coefficients of the potential real by rephasing $\Phi_{2}$.

This transformation generates off-diagonal terms $\phi_{1}^{\dagger} \phi_{2}$ and $\phi_{2}^{\dagger} \phi_{1}$ with complex coefficients given by:

$$
\frac{\left(\lambda_{1}-\lambda_{2}\right) \rho_{1} \rho_{2} e^{ \pm i \chi}}{v^{2}} .
$$

The coefficients of these bilinear terms are only real if $\sin \chi=0$ or $\lambda_{1}=\lambda_{2}$. In each case, requiring the quartic part of the potential to be also real imposes new constraints on the parameters of the potential. Therefore, confirming in this way, that in general $\mathrm{CP}$ is not conserved even if we start with a real $2 \mathrm{HDM}$ potential.

\subsection{Two non-trivial 3HDM cases}

In order to illustrate the difficulty that may arise in finding a matrix $U$ that satisfies Eq. (2.2) in a multi-doublet theory, consider the following $S_{3}$-symmetric $3 \mathrm{HDM}$ potential:

$$
V=V_{2}+V_{4}
$$

with [24, 25]

$$
\begin{aligned}
V_{2} & =\mu_{0}^{2} h_{S}^{\dagger} h_{S}+\mu_{1}^{2}\left(h_{1}^{\dagger} h_{1}+h_{2}^{\dagger} h_{2}\right), \\
V_{4} & =\lambda_{1}\left(h_{1}^{\dagger} h_{1}+h_{2}^{\dagger} h_{2}\right)^{2}+\lambda_{2}\left(h_{1}^{\dagger} h_{2}-h_{2}^{\dagger} h_{1}\right)^{2}+\lambda_{3}\left[\left(h_{1}^{\dagger} h_{1}-h_{2}^{\dagger} h_{2}\right)^{2}+\left(h_{1}^{\dagger} h_{2}+h_{2}^{\dagger} h_{1}\right)^{2}\right] \\
& +\lambda_{4}\left[\left(h_{S}^{\dagger} h_{1}\right)\left(h_{1}^{\dagger} h_{2}+h_{2}^{\dagger} h_{1}\right)+\left(h_{S}^{\dagger} h_{2}\right)\left(h_{1}^{\dagger} h_{1}-h_{2}^{\dagger} h_{2}\right)+\text { h.c. }\right]+\lambda_{5}\left(h_{S}^{\dagger} h_{S}\right)\left(h_{1}^{\dagger} h_{1}+h_{2}^{\dagger} h_{2}\right) \\
& +\lambda_{6}\left[\left(h_{S}^{\dagger} h_{1}\right)\left(h_{1}^{\dagger} h_{S}\right)+\left(h_{S}^{\dagger} h_{2}\right)\left(h_{2}^{\dagger} h_{S}\right)\right]+\lambda_{7}\left[\left(h_{S}^{\dagger} h_{1}\right)\left(h_{S}^{\dagger} h_{1}\right)+\left(h_{S}^{\dagger} h_{2}\right)\left(h_{S}^{\dagger} h_{2}\right)+\text { h.c. }\right] \\
& +\lambda_{8}\left(h_{S}^{\dagger} h_{S}\right)^{2},
\end{aligned}
$$


where all parameters are real. Here, $h_{1}$ and $h_{2}$ are members of an $S_{3}$ doublet, whereas $h_{S}$ is a singlet. These fields are related to the fields in the defining representation of $S_{3}, \phi_{i}, i=1,2,3$, by:

$$
\left(\begin{array}{l}
h_{1} \\
h_{2}
\end{array}\right)=\left(\begin{array}{c}
\frac{1}{\sqrt{2}}\left(\phi_{1}-\phi_{2}\right) \\
\frac{1}{\sqrt{6}}\left(\phi_{1}+\phi_{2}-2 \phi_{3}\right)
\end{array}\right), \quad h_{S}=\frac{1}{\sqrt{3}}\left(\phi_{1}+\phi_{2}+\phi_{3}\right) .
$$

The $S U(2)$ structure is decomposed as

$$
h_{k}=\left(\begin{array}{c}
h_{k}^{+} \\
\left(w_{k}+\eta_{k}+i \chi_{k}\right) / \sqrt{2}
\end{array}\right), \quad k=1,2, S .
$$

In addition to 11 distinct real vacua, this potential possesses 14 distinct complex vacua [26], where at least one of the parameters

$$
\left(w_{1}, w_{2}, w_{S}\right)
$$

is complex. We shall discuss two particular cases, where some vacuum expectation values are complex, and yet, CP is conserved. These will in the following be denoted C-III-c and C-IV-e [26], where the "III" and "IV" refer to the number of consistency constraints that are required for the particular vacuum.

\subsubsection{The vacuum C-III-c}

This vacuum is characterized by

$$
\left(w_{1}, w_{2}, w_{S}\right)=\left(\hat{w}_{1} e^{i \sigma_{1}}, \hat{w}_{2} e^{i \sigma_{2}}, 0\right),
$$

where $\hat{w}_{1}$ and $\hat{w}_{2}$ are real, and the three constraints are

$$
\begin{gathered}
\mu_{1}^{2}=-\left(\lambda_{1}+\lambda_{3}\right)\left(\hat{w}_{1}^{2}+\hat{w}_{2}^{2}\right), \\
\lambda_{2}+\lambda_{3}=0, \quad \lambda_{4}=0 .
\end{gathered}
$$

It is not apparent, at first sight, that this vacuum conserves $\mathrm{CP}$, mainly due to the fact that $\hat{w}_{1}$ and $\hat{w}_{2}$ are different in general. It is possible to show that CP is conserved [18], by first making a transformation to the Higgs basis [19, 20]:

$$
\left(\begin{array}{l}
h_{1}^{\prime} \\
h_{2}^{\prime} \\
h_{S}^{\prime}
\end{array}\right)=\frac{1}{v}\left(\begin{array}{ccc}
\hat{w}_{1} & \hat{w}_{2} & 0 \\
\hat{w}_{2} & -\hat{w}_{1} & 0 \\
0 & 0 & v
\end{array}\right)\left(\begin{array}{ccc}
e^{-i \sigma_{1}} & 0 & 0 \\
0 & e^{-i \sigma_{2}} & 0 \\
0 & 0 & 1
\end{array}\right)\left(\begin{array}{l}
h_{1} \\
h_{2} \\
h_{S}
\end{array}\right)
$$

with the normalization given by $v^{2}=\left(\hat{w}_{1}^{2}+\hat{w}_{1}^{2}\right)$. The coefficients of the potential remain real, so we see explicitly that $\mathrm{CP}$ is conserved.

Finding a transformation $U$ satisfying Eq. (2.2) is now possible by exploiting the fact that $\hat{w}_{S}=0$. We construct a transformation of the following form

$$
U=e^{i\left(\delta_{1}+\delta_{2}\right)}\left(\begin{array}{ccc}
\cos \theta & \sin \theta & 0 \\
-\sin \theta & \cos \theta & 0 \\
0 & 0 & 1
\end{array}\right)\left(\begin{array}{lll}
0 & 1 & 0 \\
1 & 0 & 0 \\
0 & 0 & 1
\end{array}\right)\left(\begin{array}{ccc}
\cos \theta & -\sin \theta & 0 \\
\sin \theta & \cos \theta & 0 \\
0 & 0 & 1
\end{array}\right)
$$


and choose $\theta$ such that the vevs become [18]

$$
\left(a e^{i \delta_{1}}, a e^{i \delta_{2}}, 0\right)
$$

i.e., the non-zero vevs have the same modulus. This step is based on the fact that for $\lambda_{4}=0$ the potential acquires an $S O(2)$ symmetry between $h_{1}$ and $h_{2}$. Now, an overall rephasing of the fields, which also leaves the potential invariant, given by $e^{-i\left(\delta_{1}+\delta_{2}\right) / 2}$ leads to vevs of the form

$$
\left(a e^{i \delta}, a e^{-i \delta}, 0\right) .
$$

This overall phase rotation is not felt by the vev of $h_{S}$ because it is zero. Now, due to $\lambda_{4}=0$, the potential is also symmetric under

$$
h_{1} \leftrightarrow h_{2},
$$

and one can see that CP is conserved because Eq. (2.2) can be verified by means of the matrix $U$ written above, which encodes the three steps just described. Each one of these steps is based on a symmetry property of the potential.

\subsubsection{The vacuum C-IV-e}

This case differs significantly from the previous one, since $\hat{w}_{S} \neq 0$. However, $\hat{w}_{1}$ and $\hat{w}_{2}$ are now related. In fact, the vacuum is given by

$$
\left(\sqrt{-\frac{\sin 2 \sigma_{2}}{\sin 2 \sigma_{1}}} \hat{w}_{2} e^{i \sigma_{1}}, \hat{w}_{2} e^{i \sigma_{2}}, \hat{w}_{S}\right)
$$

subject to the four constraints

$$
\begin{gathered}
\mu_{0}^{2}=\frac{\sin ^{2}\left(2\left(\sigma_{1}-\sigma_{2}\right)\right)}{\sin ^{2}\left(2 \sigma_{1}\right)}\left(\lambda_{2}+\lambda_{3}\right) \frac{\hat{w}_{2}^{4}}{\hat{w}_{S}^{2}}-\frac{1}{2}\left(1-\frac{\sin 2 \sigma_{2}}{\sin 2 \sigma_{1}}\right)\left(\lambda_{5}+\lambda_{6}\right) \hat{w}_{2}^{2}-\lambda_{8} \hat{w}_{S}^{2}, \\
\mu_{1}^{2}=-\left(1-\frac{\sin 2 \sigma_{2}}{\sin 2 \sigma_{1}}\right)\left(\lambda_{1}-\lambda_{2}\right) \hat{w}_{2}^{2}-\frac{1}{2}\left(\lambda_{5}+\lambda_{6}\right) \hat{w}_{S}^{2}, \\
\lambda_{4}=0, \quad \lambda_{7}=-\frac{\sin \left(2\left(\sigma_{1}-\sigma_{2}\right)\right) \hat{w}_{2}^{2}}{\sin 2 \sigma_{1} \hat{w}_{S}^{2}}\left(\lambda_{2}+\lambda_{3}\right) .
\end{gathered}
$$

Following the approach of Ref. [18], we transform to the Higgs basis:

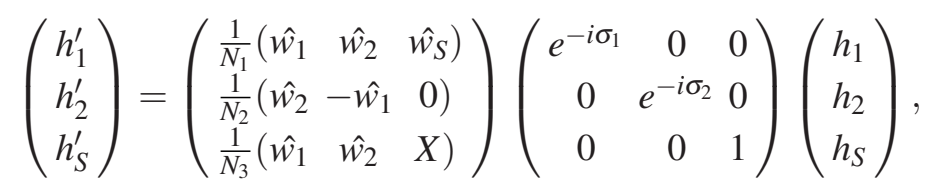

where $X$ is chosen to make lines 1 and 3 orthogonal, and with $N_{1}, N_{2}, N_{3}$ normalization factors. Performing this transformation, and making use of the freedom to perform an overall phase transformation on all fields, we see that the potential remains real, showing that $\mathrm{CP}$ is conserved.

In this example, the matrix $U$ of Eq. (2.2) is built by following two of the steps described for the vacuum C-III-c. The first step, an $S O(2)$ rotation of $h_{1}$ and $h_{2}$, corresponds to a symmetry of 
the Lagrangian because this vacuum also requires $\lambda_{4}=0$. In this case, the moduli of $w_{1}$ and $w_{2}$ are related, see Eq. (4.17). As a consequence, rotating the fields and vacuum into

$$
\left(b e^{i \gamma_{1}}, b e^{i \gamma_{2}}, \hat{w}_{S}\right)
$$

leads to

$$
\gamma_{1}+\gamma_{2}=0
$$

and an overall phase rotation is not needed in order to obtain symmetric phases for $h_{1}$ and $h_{2}$. Although the potential is symmetric under an overall phase rotation, such a rotation would make the vev of $h_{S}$ complex and would prevent Eq. (2.2) from being verified. The second step relies now on the symmetry for the interchange of $h_{1}$ and $h_{2}$.

The new matrix $U$ thus built allows for Eq. (2.2) to be verified and once again we can conclude that $\mathrm{CP}$ is not spontaneously broken.

\section{Summary}

We have discussed powerful methods that exist to check for CP conservation in multi-Higgsdoublet models, NHDM:

- For $N=2$, a full set of CP-odd invariants are established that can reveal whether or not CP is conserved.

- For $N \geq 3$ the analysis becomes more complicated and we advocate going first to the Higgs basis and checking whether or not the remaining $U(N-1)$ rotation freedom allows to transform into a potential with real coefficients.

Acknowledgements: We thank the local organizers of Corfu 2017 for the very fruitful scientific meeting and the warm hospitality. The work of PO was supported in part by the Research Council of Norway. The work of MNR was partially supported by Fundação para a Ciência e a Tecnologia (FCT, Portugal) through the projects CERN/FIS-NUC/0010/2015, and CFTP-FCT Unit 777 (UID/FIS/00777/2013) which are partially funded through POCTI (FEDER), COMPETE, QREN and EU. MNR benefited from COST support for a STSM to visit the University of Bergen under COST action CA15108 and also benefited from discussions that took place at the University of Warsaw during visits supported by the the HARMONIA project of the National Science Centre, Poland, under contract UMO-2015/18/M/ST2/00518 (2016-2019).

\section{References}

[1] J. F. Gunion, H. E. Haber, G. L. Kane and S. Dawson, “The Higgs Hunter's Guide,” Front. Phys. 80 (2000) 1 .

[2] G. C. Branco, P. M. Ferreira, L. Lavoura, M. N. Rebelo, M. Sher and J. P. Silva, "Theory and phenomenology of two-Higgs-doublet models," Phys. Rept. 516 (2012) 1 doi:10.1016/j.physrep.2012.02.002 [arXiv:1106.0034 [hep-ph]].

[3] I. P. Ivanov, "Building and testing models with extended Higgs sectors," Prog. Part. Nucl. Phys. 95 (2017) 160 doi:10.1016/j.ppnp.2017.03.001 [arXiv:1702.03776 [hep-ph]]. 
[4] K. Olaussen, P. Osland and M. A. Solberg, "Symmetry and Mass Degeneration in Multi-Higgs-Doublet Models,” JHEP 1107 (2011) 020 doi:10.1007/JHEP07(2011)020 [arXiv:1007.1424 [hep-ph]].

[5] W. Grimus and M. N. Rebelo, "Automorphisms in gauge theories and the definition of CP and P," Phys. Rept. 281 (1997) 239 doi:10.1016/S0370-1573(96)00030-0 [hep-ph/9506272].

[6] G. C. Branco, J. M. Gerard and W. Grimus, “Geometrical T Violation,” Phys. Lett. 136B (1984) 383. doi:10.1016/0370-2693(84)92024-0

[7] G. C. Branco, L. Lavoura and J. P. Silva, “CP Violation,” Int. Ser. Monogr. Phys. 103 Oxford University Press (1999).

[8] G. C. Branco, M. N. Rebelo and J. I. Silva-Marcos, "CP-odd invariants in models with several Higgs doublets,” Phys. Lett. B 614 (2005) 187 doi:10.1016/j.physletb.2005.03.075 [hep-ph/0502118].

[9] J. F. Gunion and H. E. Haber, "Conditions for CP-violation in the general two-Higgs-doublet model," Phys. Rev. D 72 (2005) 095002 doi:10.1103/PhysRevD.72.095002 [hep-ph/0506227].

[10] B. Grzadkowski, O. M. Ogreid and P. Osland, "Spontaneous CP violation in the 2HDM: physical conditions and the alignment limit," Phys. Rev. D 94 (2016) no.11, 115002 doi:10.1103/PhysRevD.94.115002 [arXiv:1609.04764 [hep-ph]].

[11] L. Lavoura and J. P. Silva, "Fundamental CP violating quantities in a SU(2) x U(1) model with many Higgs doublets,” Phys. Rev. D 50 (1994) 4619 doi:10.1103/PhysRevD.50.4619 [hep-ph/9404276].

[12] F. J. Botella and J. P. Silva, "Jarlskog - like invariants for theories with scalars and fermions," Phys. Rev. D 51 (1995) 3870 doi:10.1103/PhysRevD.51.3870 [hep-ph/9411288].

[13] B. Grzadkowski, O. M. Ogreid and P. Osland, "Measuring CP violation in Two-Higgs-Doublet models in light of the LHC Higgs data,” JHEP 1411 (2014) 084 doi:10.1007/JHEP11(2014)084 [arXiv:1409.7265 [hep-ph]].

[14] B. Grzadkowski, O. M. Ogreid and P. Osland, "Testing the presence of CP violation in the 2HDM," PoS CORFU 2014 (2015) 086 [arXiv:1504.06076 [hep-ph]].

[15] I. de Medeiros Varzielas, S. F. King, C. Luhn and T. Neder, "CP-odd invariants for multi-Higgs models: applications with discrete symmetry,” Phys. Rev. D 94 (2016) no.5, 056007 doi:10.1103/PhysRevD.94.056007 [arXiv:1603.06942 [hep-ph]].

[16] I. de Medeiros Varzielas, S. F. King, C. Luhn and T. Neder, "Spontaneous CP violation in multi-Higgs potentials with triplets of $\Delta\left(3 n^{2}\right)$ and $\Delta\left(6 n^{2}\right)$," JHEP 1711 (2017) 136 doi:10.1007/JHEP11(2017)136 [arXiv:1706.07606 [hep-ph]].

[17] S. Davidson and H. E. Haber, "Basis-independent methods for the two-Higgs-doublet model," Phys. Rev. D 72 (2005) 035004 Erratum: [Phys. Rev. D 72 (2005) 099902] doi:10.1103/PhysRevD.72.099902, 10.1103/PhysRevD.72.035004 [hep-ph/0504050].

[18] O. M. Ogreid, P. Osland and M. N. Rebelo, "A Simple Method to detect spontaneous CP Violation in multi-Higgs models,” JHEP 1708 (2017) 005 doi:10.1007/JHEP08(2017)005 [arXiv:1701.04768 [hep-ph]].

[19] J. F. Donoghue and L. F. Li, "Properties of Charged Higgs Bosons," Phys. Rev. D 19 (1979) 945. doi:10.1103/PhysRevD.19.945

[20] H. Georgi and D. V. Nanopoulos, "Suppression of Flavor Changing Effects From Neutral Spinless Meson Exchange in Gauge Theories,” Phys. Lett. 82B (1979) 95. doi:10.1016/0370-2693(79)90433-7 
[21] T. D. Lee, “A Theory of Spontaneous T Violation,” Phys. Rev. D 8 (1973) 1226. doi:10.1103/PhysRevD.8.1226

[22] G. C. Branco and M. N. Rebelo, "The Higgs Mass in a Model With Two Scalar Doublets and Spontaneous CP Violation,” Phys. Lett. 160B (1985) 117. doi:10.1016/0370-2693(85)91476-5

[23] J. F. Gunion and H. E. Haber, "The CP conserving two Higgs doublet model: The Approach to the decoupling limit,” Phys. Rev. D 67 (2003) 075019 doi:10.1103/PhysRevD.67.075019 [hep-ph/0207010].

[24] E. Derman, "Flavor Unification, $\tau$ Decay and $b$ Decay Within the Six Quark Six Lepton Weinberg-Salam Model,” Phys. Rev. D 19 (1979) 317. doi:10.1103/PhysRevD.19.317

[25] D. Das and U. K. Dey, “Analysis of an extended scalar sector with $S_{3}$ symmetry,” Phys. Rev. D 89 (2014) no.9, 095025 Erratum: [Phys. Rev. D 91 (2015) no.3, 039905] doi:10.1103/PhysRevD.91.039905, 10.1103/PhysRevD.89.095025 [arXiv:1404.2491 [hep-ph]].

[26] D. Emmanuel-Costa, O. M. Ogreid, P. Osland and M. N. Rebelo, "Spontaneous symmetry breaking in the $S_{3}$-symmetric scalar sector,' JHEP 1602 (2016) 154 Erratum: [JHEP 1608 (2016) 169] doi:10.1007/JHEP08(2016)169, 10.1007/JHEP02(2016)154 [arXiv:1601.04654 [hep-ph]]; D. Emmanuel-Costa, O. M. Ogreid, P. Osland and M. N. Rebelo, "CP Violation in the scalar sector," PoS CORFU 2015 (2016) 044 [arXiv:1604.00637 [hep-ph]]. 